\title{
Importance of Oral Health in Children
}

\author{
Hernández Mendieta LA ${ }^{1}$ and Lara Carrillo E ${ }^{2 *}$ \\ ${ }^{1}$ School of Dentistry, Autonomous University of Mexico State, Mexico \\ ${ }^{2}$ Professor and Dean of the School of Dentistry, Autonomous University of Mexico State, \\ Mexico
}

*Corresponding author: Edith Lara-Carrillo, Professor and Dean of the School of Dentistry, Autonomous University of Mexico State, Toluca, C.P. 50130, México, Tel: 527221582062; Email: elarac@uaemex.mx

Keywords: Dental Caries; Temporary Dentition; Oral Health

\section{Etiology}

Caries is a multifactorial disease, a high sugar diet and poor oral hygiene can cause a state of oral dysbiosis, which will lead to demineralization of the teeth and consequently carious lesions [1]. In Dentistry, dental caries is one of the main problems in oral health, in fact it is creating a challenge for clinicians due to the responsibility of providing adequate treatment based on scientific evidence to patients, in this case pediatric patients, because if carious lesions are not treated on time, they will evolve into a pulp pathology, generating pain and low quality of life related to patient's health [2]. In pediatric patients the history of pain is unclear because symptoms can start from a reversible stage and a favorable prognosis to an irreversible stage of the dental pulp. In addition is not directly the patient who provide the symptoms, instead are the parent who refer to child's feedback, and most of the time is only the pain the reason to visit the dentist $[3,4]$. Since the middle of the 20th century, the need for endodontic treatment has increased, that means the total or partial removal of the dental pulp; because it has been studied how the condition of the pulp affects the development of infectious processes [5]. It is estimated that $90 \%$ of pediatric patients seeking for dental care are affected by dental pulp symptoms. Therefore, pediatric dentists are obliged to know the frequency of appearance and the indications for treatment in pulp pathologies of primary teeth, when the diagnoses are made on time, and are accompanied by correct therapy, it will prevent the progression of the disease and therefore the complications [5].

\section{Epidemiology}

For several decades, various researchers [6-9] have emphasized the importance of taking care of the deciduous dentition to improve the oral health in the permanent dentition. Dental caries is considered a public health problem due to its high prevalence in pediatric patients throughout the world, affecting approximately $9 \%$ of the world children [10]; between the principal risk factors is sociocultural and socioeconomic background of parents, among others $[1,11]$. It has been found that the average prevalence of caries in children under 2 years old is $17 \%$, and this increases with age; at the age of 2 exist a prevalence of $36 \%$, with 3,4 and 5 years old the prevalence increases to $43 \%, 55 \%$ and $63 \%$ respectively, leading to the conclusion that not only the prevalence increases with age, also in younger patients than 3 years old who do not receive the necessary care this disease progresses [12].

It has been described that patients with carious lesions in primary teeth have 5 to 6 times higher incidence of developing new lesions in permanent dentition, compared to caries-free patients at early age [1]. de Campos Mello TR, et al. [11] mention that being black or brown, attending school in rural areas, and attending public school were identified as determining factors for a higher probability of presenting deciduous teeth with untreated cavities. Being a female was identified as a protective factor. Carious lesions not only affect oral health, but they also affect general health and quality of life, causing not only dental pain, but also dental enamel defects, eating problems due to an improper diet, speaking problems, difficulty sleeping, decreased school performance, low self-esteem, poor social relationships, and an increased risk of developing carious lesions in permanent 


\section{Epidemiology International Journal}

dentition $[1,10,13,14]$.

\section{Timely Care}

Unfortunately, many of the causes of lack to attend primary dentition are due to parents' misinformation, considering that they are teeth that are going to fall out and it is not a permanent dentition; therefore, they do not consider the need for timely care, in addition to adverse sociodemographic factors and ignorance of the importance of oral health. Untreated carious lesions are directly associated with the quality of life of the patient and the family, especially severe carious lesions $[14,15]$. Patients who have been treated on time with conventional and biological treatments remain asymptomatic during the following 6-years; this high success rate contributes to the improvement in the general state of health and quality of life of patients. Recent research has concluded that it is not known with certainty when the injuries begin to have a negative impact on the quality of life of the pediatric patient, so it is of great importance to treat patients in a timely manner [14,15]. Likewise, it is important to have epidemiological information on the diversity of the population, as it will be extremely useful for planning dental care strategies and establish protocols for action in local health systems, contributing to equal access and health care services.

\section{References}

1. Meyer F, Enax J (2018) Early Childhood Caries: Epidemiology, aetiology, and prevention. Int J Dent 1415873.

2. Kassa D, Day P, High A, Duggal M (2009) Histological comparison of pulpal inflammation in primary teeth with occlusal or proximal caries. Int J Paediatr Dent 19(1): 26-33.

3. Fuks AB, Kupietzky A, Guelmann M (2020) Pulp therapy for the primary dentition. In: American Academy of Pediatric Dentistry. Pulp therapy for primary and immature permanent teeth. The Reference Manual of Pediatric Dentistry, American Academy of Pediatric Dentistry, Chicago, Ill, USA, pp: 384-392.

4. Dhar V, Marghalani AA, Crystal YO, Kumar A, Ritwik P, et al. (2017) Use of vital pulp therapies in primary teeth with deep caries lesions. Pediatr Dent 39(5): 146-159.

5. Damyanova DM, Angelova S, Borisova RA (2018) Estimation of pulpitis prevalence in primary dentition. Dent Res Oral Health 1(3): 29-33.

6. Ghezzi F, Zallio F, Mazzarello GP, Tampelloni C (1986) Epidemiological investigation on the incidence of caries and malocclusions of the deciduous teeth in children of nursery schools of the USL 16 (Genoa-Levante). Minerva Stomatol 35(3): 107-112.

7. Castellani G, Bertelè GP, Zerman N (1987) Epidemiologic study in nursery schools of the Verona Commune on the occurrence of dental caries, malocclusion and bad habits that can influence the normal development of facial bone structure in children. Minerva Stomatol 36(3):121-125.

8. Milicić A, Coklica VG, Hunski M (1984) An analysis of the incidence of orthodontic anomalies and caries in the deciduous teeth of children from Zagreb. Acta Stomatol Croat 18(2): 95-103.

9. Melsen B, Terp S (1982) The influence of extractions caries cause on the development of malocclusion and need for orthodontic treatment. Swed Dent J Suppl 15: 163-169.

10. Faria PC, Daher A, Freire MDCM, de Abreu MHNG, Bonecker $\mathrm{M}$, et al. (2018) Impact of untreated dental caries severity on the quality of life of preschool children and their families: a cross-sectional study. Qual Life Res 27(12): 3191-3198.

11. de Campos Mello TR, Antunes JL, Waldman EA (2008) Prevalence of untreated caries in deciduous teeth in urban and rural areas in the state of São Paulo, Brazil. Rev Panam Salud Publica 23(2): 78-84.

12. Tinanoff N, Baez RJ, Guillory CD, Donly KJ, Feldens CA, et al. (2019) Early childhood caries epidemiology, aetiology, risk assessment, societal burden, management, education, and policy: Global perspective. Int J Paediatr Dent 29(3): 238-248.

13. Masumo RM, Ndekero TS, Carneiro LC (2020) Prevalence of dental caries in deciduous teeth and oral health related quality of life among preschool children aged 4-6 years in Kisarawe, Tanzania. BMC Oral Health 20(1): 46.

14. Banihani A, Deery C, Toumba J, Munyombwe T, Duggal M (2017) The impact of dental caries and its treatment by conventional or biological approaches on the oral health-related quality of life of children and carers. Int J Paediatr Dent 28(2): 266-276.

15. Jorge JR, Pordeus IA, Ramos Jorge ML, Marques LS, Paiva SM (2014) Impact of untreated dental caries on quality of life of preschool children: different stages and activity. Community Dent Oral Epidemiol 42(4): 311-322. 